\title{
QUEEN'S
UNIVERSITY
BELFAST
}

\section{Resource misallocation as a mediator of fitness costs in antibiotic resistance}

Trauner, A., Banaei-Esfahani, A., Gygli, S. M., Warmer, P., Feldmann, J., Shafieechashmi, S., Eschbach, K., Zampieri, M., Borrell, S., Collins, B. C., Beisel, C., Aebersold, R., \& Gagneux, S. (2018, Oct 30). Resource misallocation as a mediator of fitness costs in antibiotic resistance. https://doi.org/10.1101/456434

\section{Document Version:}

Early version, also known as pre-print

Queen's University Belfast - Research Portal:

Link to publication record in Queen's University Belfast Research Portal

\section{Publisher rights}

Copyright 2017 The Authors. This is an open access article published under a Creative Commons Attribution License (https://creativecommons.org/licenses/by/4.0/), which permits unrestricted use, distribution and reproduction in any medium, provided the author and source are cited.

\section{General rights}

Copyright for the publications made accessible via the Queen's University Belfast Research Portal is retained by the author(s) and / or other copyright owners and it is a condition of accessing these publications that users recognise and abide by the legal requirements associated with these rights.

Take down policy

The Research Portal is Queen's institutional repository that provides access to Queen's research output. Every effort has been made to ensure that content in the Research Portal does not infringe any person's rights, or applicable UK laws. If you discover content in the Research Portal that you believe breaches copyright or violates any law, please contact openaccess@qub.ac.uk. 


\section{Resource misallocation as a mediator of fitness costs}

\section{in antibiotic resistance.}

Andrej Trauner ${ }^{1,2, *}$, Amir Banaei-Esfahani, ${ }^{3,4}$, Sebastian Gygli, ${ }^{1,2}$, Philipp Warmer ${ }^{3}$, Julia

Feldmann ${ }^{1,2}$, Seyedehsara Shafieechashmi ${ }^{1,2}$, Katja Eschbach ${ }^{5}$, Mattia Zampieri ${ }^{3}$, Sonia Borrell ${ }^{1,2}$,

Ben C. Collins ${ }^{3}$, Christian Beisel ${ }^{5}$, Ruedi Aebersold ${ }^{3,6}$ and Sebastien Gagneux ${ }^{1,2}$

${ }^{1}$ Swiss Tropical and Public Health Institute, Basel, Switzerland

${ }^{2}$ University of Basel, Basel, Switzerland

${ }^{3}$ Department of Biology, Institute of Molecular and Systems Biology, ETH Zurich, Zurich, Switzerland

${ }^{4} \mathrm{PhD}$ Program in Systems Biology, Life Science Zurich Graduate School, University of Zurich and ETH Zurich, Zurich, Switzerland

${ }^{5}$ Genomics Facility Basel, Department of Biosystems Science and Engineering, ETH Zurich, Basel, Switzerland

${ }^{6}$ Faculty of Science, University of Zurich, Zurich, Switzerland

*andrej.trauner@swisstph.ch 


\section{Abstract}

Antimicrobial resistance (AMR) poses a threat to global health and the economy. Rifampicinresistant Mycobacterium tuberculosis accounts for a third of the global AMR burden. Gaining the upper hand on AMR requires a deeper understanding of the physiology of resistance.

AMR often results in the erosion of normal cell function: a fitness cost. Identifying intervention points in the mechanisms underpinning the cost of resistance in $M$. tuberculosis could play a pivotal role in strengthening future treatment regimens. We used a collection of $M$. tuberulosis strains providing an evolutionary and phylogenetic snapshot of rifampicin resistance and subjected them to genome-wide transcriptomic and proteomic profiling to identify key perturbations of normal physiology.

We found that a rifampicin resistance-conferring mutation in RpoB imparts considerable gene expression changes, many of which are mitigated by a compensatory mutation in RpoC.

However, our data also provide evidence for pervasive epistasis: the same resistance mutation imposed a different fitness cost and functionally unrelated changes to gene expression in clinical strains from unrelated genetic backgrounds. Rather than functional changes in specific pathways, our data suggest that the fitness cost of rifampicin resistance stems from a misallocation of resources: the greater the departure from the wild type baseline proteome investment, the greater the fitness cost of rifampicin resistance in a given strain. We summarize these observations in the "Burden of Expression" hypothesis of fitness cost and provide evidence that it can be used for suppressing the emergence of rifampicin resistance. 


\section{Introduction}

Antimicrobials are one of the cornerstones of modern medicine ${ }^{1}$. The global increase of antimicrobial resistance (AMR) poses an existential threat, claiming an increasing number of lives and resources ${ }^{2}$. We currently have access to a wide array of antibiotics, but their efficacy is waning, making safeguarding existing and future drugs a high priority. Understanding the mechanisms and drivers of $\mathrm{AMR}^{3}$, including the underlying biology, will be key to that process. Antibiotics target essential bacterial processes. Modification of their targets is an important mechanism through which AMR emerges. It is therefore not surprising that AMR often comes with a fitness $\operatorname{cost}^{4}$. Fitness cost is a broad concept capturing any negative deviation in the proliferation of a mutant from its ancestor: for example, a decreased growth rate in vitro, or in the case of pathogens, a decreased ability to transmit or cause disease. The physiological basis for the cost of drug resistance seems to be dependent on the antibiotic, bacterial species and environment ${ }^{5}$ and is thus often unknown and likely to be multifaceted. One of the better studied examples is the cost of rifampicin resistance. Rifampicin targets the bacterial RNA polymerase (RNAP), and resistance to rifampicin is usually mediated by mutations in the $\beta$ subunit of $\mathrm{RNAP}^{6}$. Several studies point to the rate of transcription, particularly as it pertains to the synthesis of ribosomal RNA and ribosomal proteins, as an important mediator of growth rate . $^{7,8}$. A slowing down of transcription is therefore the prime mechanistic candidate for the cost of rifampicin resistance ${ }^{9,10}$. The mechanism linking RNAP activity to ribosome biosynthesis provides a compelling explanation for the cost of rifampicin resistance in rapidly dividing bacteria such as Escherichia coli and Pseudomonas aeruginosa whose growth relies on the rapid replenishment of biosynthetic machinery lost through cell division ${ }^{11}$. Importantly, the fitness cost of rifampicin resistance can be mitigated or even reversed through the acquisition of secondary, compensatory mutations in the $\alpha, \beta$ and $\beta^{\prime}$ subunits of RNAP that seem to restore normal enzyme function ${ }^{9,12,13}$.

Rifampicin-resistant $M t b$ is one of the major causes of AMR-associated mortality globally, claiming an estimated 240,000 lives in $2016^{14}$, and unlike in fast-growing bacteria, the rate of 
transcription does not seem to reflect the fitness cost of key rpoB mutations, measured either as growth rate in vitro or prevalence in the clinic $^{13,15,16}$. While relative fitness does seem to determine the clinical success of rifampicin-resistant $M t b^{17}$, and compensatory mutations are frequently found in settings with a high burden of drug resistant $\mathrm{TB}^{18-21}$, the basis for the fitness cost of rifampicin resistance remains unknown in $M t b$. Understanding the mechanism by which $\not p o B$ mutations impair normal Mtb physiology could help identify new intervention points, through which we could stem the tide of existing and emergent rifampicin resistance.

We used the known ability of mutations in the beta barrel double $\psi$ (BBDP) domain of the $\beta$ ' subunit of RNAP to compensate for the fitness cost of resistance mutations occurring in the $\beta$ subunit in $M t b$ as a starting point ${ }^{12,13,22}$. Compensatory mutations improve patient to patient transmission of rifampicin-resistant strains ${ }^{19}$, and partially reverse biochemical changes imparted on RNAP by rifampicin-resistance mutations ${ }^{12,13}$. We hypothesise that the same would be true for gene expression differences. Leveraging the knowledge of the role of RpoC mutations, we used transcriptomic and proteomic expression profiling to identify the signature of compensation and therefore infer the likely mediators of fitness cost in a collection of strains derived from a drugsusceptible clinical isolate (see Figure 1). Our findings point to the idiosyncratic consequences of expressional dysregulation as a key factor conferring a fitness cost to rifampicin resistance in $M t b$. We expanded on this observation by profiling the expression signature of rifampicin resistance in a panel of genetically diverse clinical isolates sharing the same rifampicin resistance-conferring mutation: RpoB Ser450Leu. While we found very little evidence for a shared expression signature of rifampicin-resistance across the tested strain pairs, we show a correlation between the fitness cost of the rifampicin-resistance conferring mutation and the extent to which its presence imparts a deviation from the proteome composition of the wild-type. Finally, we show that this correlation could be exploited to suppress the emergence of rifampicin resistance. 


\section{Results}

\section{Compensatory mutations mitigate resistance-imposed expression changes}

Physiological changes incurred by a fitness cost are likely to manifest as deviations in gene expression. Since mutations in the BBDP domain of the $\beta$ ' subunit of RNAP mitigate the fitness cost of rifampicin-resistance mutations in $M t b^{12,13,22}$ they should also impact and therefore highlight expression changes that are relevant to the understanding of fitness cost of rifampicin resistance.

We previously reported the result of a directed evolution experiment in which we identified a mutation in the BBDP domain: RpoC Leu516Pro as a putative compensatory mechanism for the fitness cost of the rifampicin-resistance conferring mutation RpoB Ser450Leu in a clinical isolate $^{18}$. The strains generated by that study comprise the original drug-susceptible isolate (DS), its laboratory-derived rifampicin-resistant mutant (RpoB Ser450Leu, RifR) and the resulting evolved strains obtained by serial passage in the absence of rifampicin for 200 generations (DS ${ }^{\text {evo }}$ and RifR ${ }^{\text {evo }}$, respectively, see Figure 1A). Together these strains offer a representative snapshot of the evolutionary process that passes through the initial emergence of (costly) drug resistance and leads to the establishment of a mature drug-resistant strain whose fitness is indistinguishable from its drug susceptible ancestor. We therefore hypothesised that comparative transcriptomic and proteomic expression profiling of these strains will allow us to determine the signature of the fitness cost associated with rifampicin resistance.

First, we determined the relative fitness of RifR. Using a mixed effect linear regression model to analyse growth assays, we noted a $26.4 \%$ decrease $\left(\mathrm{CI}^{95 \%}: 21.5-31.0 \%, \mathrm{p}<0.001\right)$ in the growth rate of RifR when compared to DS. The comparison of their evolved counterparts - DS $^{\text {evo }}$ and RifR $^{\text {evo }}-$ showed no significant differences $\left(-1.2 \%, \mathrm{CI}^{95 \%}:-10.8-7.1 \%, \mathrm{p}=0.814\right)$, illustrating the fact that RpoC Leu516Pro does indeed compensate the fitness cost of rifampicin resistance. 
We aimed to identify differences in the baseline, unperturbed, gene expression as a proxy for describing the biological basis for reduced fitness in RifR. We sampled actively growing bacterial cultures of each of the four strains, extracting total RNA and protein to be profiled using RNA sequencing (RNAseq) and sequential window acquisition of all theoretical mass spectra (SWATH-MS), respectively (see Figure 1B). In total, we were able to obtain RNA transcript counts for all present regions of the $M t b$ genome and reliably quantify 2,886 proteins across our samples (Supplementary Figure 1). We used differential expression analysis to test our hypothesis that the compensatory mutation RpoC Leu516Pro had the net effect of reversing, at least partially, the expression changes brought about by the rifampicin resistance mutation RpoB Ser450Leu. We named this trend a "signature of compensation" - see Figure $2 \mathrm{~A}$ and we derived it by identifying genes that are uniquely differentially expressed in RifR compared to the other three strains in our dataset. To maximise the probability of identifying the signature of compensation, we chose an inclusive definition of differential expression: a p-value of less than 0.05 after adjusting for multiple testing (see Methods). In keeping with our inclusive approach, we also deliberately did not use an effect size threshold (e.g. minimum log-fold change).

Using these criteria, we identified 536 transcripts that could be involved in the cost of resistance. 289 transcripts were less abundant and 247 were more abundant in RifR compared to the other samples. Similarly, 536 proteins showed a significant signature of compensation: 260 proteins were more and 276 were less-abundant in RifR (see Figure 2B). Gene set enrichment analysis of the transcriptomic and proteomic data pointed to iron homeostasis being significantly affected. Specifically, it indicated a higher expression, in RifR, of genes that are repressed by the irondependent regulator (IdeR, Rv2711) in iron replete conditions. Among them, there was a significant enrichment of genes involved in polyketide and non-ribosomal peptide synthesis, which include the biosynthetic machinery for the sole $M t b$ siderophore: mycobactin (see Supplementary Figure 2-4). These changes suggested that RifR faced a shortage of iron in our experimental conditions. 
The availability of iron is an essential requirement for $M t b$ growth, both in culture and during infection, and iron acquisition systems are therefore key virulence factors ${ }^{23-25}$. Hence, an increased requirement for iron could manifest itself as a loss of relative fitness. The fact that RpoB

Ser450Leu led to a modification of the expression of genes involved in iron homeostasis and that RpoC Leu516Pro reversed the effect provides a compelling alternative mechanism underpinning the apparent fitness cost of rifampicin resistance. If the disruption of iron homeostasis drives fitness cost, we would expect that iron supplementation should mitigate the relative cost of RpoB Ser450Leu. Furthermore, based on the expression profile, we expected that RifR should produce more mycobactin at baseline than DS, potentially influencing the overall growth rate of the mutant.

We addressed the first hypothesis by comparing growth rates of RifR and DS in the presence or absence of $10 \mu \mathrm{M}$ hemin - an additional source of iron that is by itself sufficient to support the growth of a mutant defective in mycobactin biosynthesis. Importantly, hemin and mycobactin provide two separate routes of iron uptake, which allows us to side-step issues that might emerge from deficient iron transport ${ }^{23}$. The presence of hemin did not change the cost of RifR, which we calculated to be $18.6 \%$ in the absence and $20.9 \%$ in the presence of hemin for this experiment (Mixed effect linear model, $\mathrm{p}=0.737)$. Similarly, hemin did not impact the growth rate of DS ($\left.4.7 \%, \mathrm{CI}^{95 \%}:-16.3-2.3 \%, \mathrm{p}=0.128\right)$. In summary, iron did not appear to limit the growth of RifR under normal conditions.

Next, we addressed the production of mycobactin. We prepared whole cell extracts from DS and RifR grown in both, normal medium and medium supplemented with $10 \mu \mathrm{M}$ hemin. We found that on average RifR produced more mycobactin than DS, corroborating the physiological relevance of the increased baseline expression of mycobactin biosynthesis genes. We also observed a slight decrease in the production of mycobactin in bacteria grown in the heminsupplemented medium, pointing to a modification of the expression of mycobactin biosynthesis cluster in response to iron (See Figure 3). Given that the growth rate was not affected by the 
presence of hemin, these findings suggest that mycobactin itself does not modulate the growth rate of the mutant. It is therefore possible that the higher expression of the biosynthetic cluster itself might impart a fitness cost.

Interestingly, while significantly enriched, only half of the genes reported to be repressed by $\mathrm{IdeR}^{26}$ in iron-replete conditions were part of the signature of compensation ( 22 out of 40 genes). This prompted us to take a closer look at the IdeR regulon and its regulation. We took advantage of recent studies modelling the global gene regulation in $M t b^{27-29}$. We reconstructed the genomewide gene regulatory network and extracted the immediate neighbours of IdeR- and ironresponsive genes ${ }^{28}$. There were 7 expression modules that contained at least 3 genes that are part of the IdeR regulon (Figure 3, black diamonds). Together, these modules covered $82.5 \%$ of all the IdeR-repressed genes, and with the exception of Module 4 (Figure 3), none of the modules included IdeR-independent iron-responsive genes. All the genes that we identified as candidates for compensation belonged to Modules 1-4, while none of the genes included in the other modules were found to be differentially expressed in RifR. A key difference among modules was that IdeR-regulated genes represented more than half of all the genes in modules affected by compensation but fewer than half in those that were not part of the "signature of compensation". Mapping proteomic data onto the same expression network produced similar results (see Supplementary Figure 5). Interestingly, few of the IdeR-independent iron-responsive genes were part of the signature of compensation. This pattern implies a modulation of the canonical function of IdeR, either through regulatory inputs from other transcription factors, or some other mechanism.

These results supported our hypothesis that mutations in rpoB impart changes to the baseline expression profile of $M t b$ that could be reversed in the presence of a compensatory mutation in $r p o C$. Combining the expression data with our findings that iron supplementation and mycobactin levels did not affect RifR growth rates, we concluded that the transcriptional changes were not driven by the demand for iron. Instead, these changes might be a reflection of a dysfunction of 
RNAP - e.g. differences in promoter specificity or modified interaction with IdeR, whose downstream consequences may impose a fitness effect. For example, as the mycobactin biosynthesis cluster comprises several large proteins, their excessive production could represent a drain on the cell's resources. If true, we would expect such effects to be universal across all $M t b$ strains carrying this $r p o B$ mutation.

\section{The impact of RpoB Ser450Leu is shaped by epistasis}

We wanted to test the hypothesis that higher expression of the mycobactin biosynthetic cluster is a general feature of rifampicin resistance in $M t b$ and therefore the underlying cause of its fitness cost. To do so, we generated RpoB Ser450Leu mutants in five genetically diverse clinical isolates belonging to two different $M t b$ lineages and profiled them. Globally, Mtb can be grouped into seven distinct genetic lineages each with a specific geographic distribution ${ }^{30} . M t b$ lineages can differ in their interaction with the human host, the dynamics of disease progression, and also in their apparent propensity to acquire drug resistance ${ }^{31,32}$. We chose strains belonging to Lineage 1 and 2, because of their large phylogenetic separation (see Supplementary Figure 6) and more importantly, because drug resistance is often associated with Lineage 2 and relatively rare in Lineage $1^{33}$. We expected that the comparison of the transcriptome and proteome between the Ser450Leu mutants and their cognate wild type ancestor would allow us to identify general patterns of fitness cost linked to this mutation.

It is important to note that this comparison did not include any compensated strains, i.e. strains carrying mutations in the BBDP domain. We were therefore unable to focus our analysis exclusively on genes whose expression was corrected by the presence of an $r p o C$ mutation. Nonetheless, direct comparison of RifR and DS is virtually indistinguishable from the signature of compensation when considering IdeR-regulated genes and therefore serves as a reasonable proxy for our analyses (see Supplementary Figure 5). 
We started by measuring the growth characteristics of the wild type isolates and the relative cost of the RpoB Ser450Leu mutation in the different strain backgrounds. The generation time varied from $22.7 \mathrm{~h}\left({ }^{95 \%} \mathrm{CI}: 20.8-25.0 \mathrm{~h}\right)$ to $31.0 \mathrm{~h}\left({ }^{95 \%} \mathrm{CI}: 29.3-35.1 \mathrm{~h}\right)$. The relative fitness cost of the RpoB Ser450Leu mutation differed as well, from a modest $2 \%$ (mixed effect linear regression, $\mathrm{p}$ $=0.71)$ to a pronounced $27 \%\left(\right.$ mixed effect linear regression, $\left.\mathrm{p}=5.6 \times 10^{-6}\right)$.

We obtained the expression profiles for each strain to check whether the pattern we identified for IdeR-repressed genes was a universal phenotype for RpoB Ser450Leu mutants. Analysing the transcriptomic data by performing a single comparison across the five strain pairs, we found that only $17.5 \%$ (7/40 genes) of the IdeR-repressed genes were significantly differentially expressed. A single gene belonging to the mycobactin biosynthesis cluster was included in that number. Proteomic analysis revealed a similar result $-17.1 \%$ (6/35 detected proteins) were found to be significantly differentially expressed across all strains, none of which belonged to the mycobactin biosynthesis cluster. None of the iron-homeostasis gene sets highlighted in the "signature of compensation" were significantly differentially expressed across all strains. Since these findings were contrary to our expectations, we stratified the analysis and mapped the differential expression results for each strain onto the IdeR- and iron-responsive gene network we collated earlier. These results echoed our combined analysis: the signature of compensation was not universal across the tested strains. N0155, which corresponds to "DS", is the only strain to show a transcriptional profile consistent with the signature of compensation (see Figure 4A). Proteomic data corroborated this finding (see Supplementary Figure 7). It is important to note that these data represent an independent replication of the experiments, from which we derived the signature of compensation, showing that our original results are robust and reproducible. However, the absence of a coherent IdeR-responsive phenotype was clear evidence of epistasis and raised a broader question: are there any commonalities in the phenotypic manifestation of the RpoB Ser450Leu mutation among our set of strains? 
To address this question we sought to identify expression modules ${ }^{28}$ whose membership was well represented among significantly differentially expressed genes in at least one pair-wise comparison between a rifampicin-resistant strain and its cognate drug-susceptible ancestor (see Methods for details). Using transcriptomic and proteomic data, we identified 33 expression modules that fitted our criterion (see Figure 4B). There was virtually no consensus across the strains in the transcriptional or translational response to the $p_{0} B$ mutation. The only case where we observed partial agreement across genetic backgrounds concerned some of the modules controlled by the hypoxia-responsive regulator $\operatorname{Dos}^{34}$. As with modules containing IdeR ironrepressed genes, we observed only partial regulon induction for DosR. Specific modules were clearly involved in the expression changes (either protein or transcript) in each background, but the impact of these was strain-specific. A complementary manifestation of this phenomenon comes from the global comparison of all rifampicin-resistant strains against all wild type strains, which highlighted a single module as enriched for significantly differentially expressed genes. Comparing the distribution of the effect sizes, as measured by the per-gene fold-changes in expression in the combined analysis and the pairwise comparisons for each strain, we saw a marked muting of the magnitude of differential expression in the former (see Supplementary Figure 8). This was likely due to the averaging effect of the combined analysis suppressing the contribution of the differential expression from individual strains. The magnitude of the expression change in pairwise comparisons was comparable across strains.

Overall, we were able to identify a wealth of gene expression changes in our samples: as many as 958 transcripts and 1914 proteins were observed to be differentially expressed in at least one comparison across our samples. On the level of individual genes, the transcriptome and to lesser extent the proteome of each strain were perturbed in their own private way (see Supplementary Figures 9\&10), manifesting itself as the drug resistance iteration of the Anna Karenina principle ${ }^{35}$. Because the majority of those changes were specific to individual strains they were largely invisible if the comparison was made across all strain pairs. The fact that the same mutation can 
have such profoundly different outcomes depending on the genetic context in which it occurs, is clear evidence of epistasis, and shows that natural genetic variation can fundamentally impact the physiological consequences and therefore evolution of drug resistance. Importantly, the impact of resistance on the expression profile of any two strains was found to be independent of the genetic distance between them (see Supplementary Figure 11).

So far, we showed that the RpoB Ser450Leu causes a considerable re-organization of baseline gene expression, that this perturbation can be reversed by a compensatory mutation in RpoC and that the specific phenotypic manifestation was dependent on mutations that occurred more recently than those defining individual lineages. These findings were consistent with our observation that the same mutation imposed a different fitness cost to different strains. We therefore sought to find correlates of the varying fitness costs.

\section{Deviation from baseline expression correlates with the cost of rifampicin resistance}

Pleiotropic phenotypes of the kind described above are not normally addressed, however we wanted to explore whether the extent of the expression perturbations correlated with the varying fitness costs of Ser450Leu we observed in different genetic backgrounds. We reasoned that the cumulative impact on expression disruption, rather than the dysregulation of individual genes, would provide a conduit for a loss of fitness.

In the first instance, we considered the correlation between the fitness cost of the $r p o B$ mutation and the overall expression distance between the mutant and its cognate wild type strain (See Supplementary Figure 12). Through this approach, we were able to detect a relationship between cost and expression differences for the expressed proteins $\left(\mathrm{R}^{2}=0.83, \mathrm{p}=0.031\right.$, ordinary least squares linear regression) but not RNA $\left(\mathrm{R}^{2}=0.39, \mathrm{p}=0.258\right.$, ordinary least squares linear regression). Given that the correlation was stronger in the proteome compartment, and that the proteome compartment seemed more affected by resistance, we elaborated on our observation by incorporating a measure of physiological cost for each protein. We used two different metrics 
for cost. In the simpler case we used the molecular weight of amino acids as proxy for the resource investment necessary to generate each protein ${ }^{36}$. We also used estimates of ATP cost for each amino acid in E. coli as a way to approximate the level of energy investment a bacterial cell makes when synthesising its proteome ${ }^{37}$. Both metrics showed that drug resistance imposes an additional physiological cost to the baseline proteome (Molecular Weight: Mann-Whitney U-test, $\mathrm{p}=8.26 \times 10^{-4}$, ATP equivalents: Mann-Whitney U-test, $\mathrm{p}=4.50 \times 10^{-4}$, see Supplementary Figure 13). Furthermore, this cost was negatively correlated with the relative fitness of the RpoB Ser450Leu mutation in a given strain background $\left(\varrho_{s}=-0.90, \mathrm{p}=0.04\right)-$ the greater the deviation from the resource investment of the ancestral proteome, the larger the cost of the mutation (see Figure 5A). Growth rate and gene expression are not independent from each other. To test the possibility that the observed correlation may be an artefact of our analysis, we took advantage of the natural variation in growth rates of different drug-susceptible clinical isolates in our medium and compared them to the relative costs of expression (See Supplementary Figure 14). We performed a pairwise comparison across all the tested strains and observed no statistically significant correlation between the differences in the investment into the proteome and the difference in growth rates $\left(\varrho_{\mathrm{s}}=0.34, \mathrm{p}=0.33\right)$. The differences in the allocation of resources into the protein compartment of different bacterial strains were therefore not the main determinant of variation in their respective generation times.

Taken together, our results seemed to suggest that the ultimate manifestation of the disruption of wild type baseline gene expression by RpoB Ser450Leu was a net increase in the biosynthetic input required to maintain the steady state proteome: the greater the cost of the disruption, the greater the slowing down of growth in a given strain background. We propose this as the "Burden of Expression" hypothesis of the fitness cost of rifampicin resistance.

\section{Carbon allocation rather than ATP availability modulates cost of resistance}

An implication of the "Burden of expression" hypothesis is the possibility of suppressing the emergence of rifampicin-resistance in mycobacteria by maximising the additional biosynthetic 
cost imposed by the deviation from the baseline expression. We tested two types of conditions that may impose such a stress: inhibition of ATP synthesis and variation of carbon-source quality. The first would disrupt the ability to generate energy through catabolic processes, while the second would place more emphasis on the anabolic aspects of bacterial growth. In the first instance, we tested the susceptibility to bedaquiline, an ATP synthase inhibitor that leads to a decrease in intracellular ATP levels in $M t b^{38}$. Given the higher baseline cost of their proteome, we expected that RpoB Ser450Leu mutants should show an increased susceptibility to bedaquiline commensurate with their relative loss of fitness. We did not observe any correlation between bedaquiline susceptibility and the cost of the RpoB Ser450Leu mutation (see Figure 5B).

Next, we explored varying carbon source quality, expecting substrates that force the bacterial cell to rely more heavily on anabolic processes to serve as amplifiers for the perceived cost of rifampicin resistance. A related phenotype has been reported before for RpoB Ser450Leu ${ }^{12}$. We chose the Luria-Delbrück fluctuation assay as an unbiased readout for the overall increase in the cost of rifampicin-resistance, because its frequency of resistance estimate contains a signal for the ability of drug resistant bacteria to propagate within the population prior to antibiotic exposure ${ }^{39}$. The global increase in the cost of RpoB mutations would therefore manifest itself as an apparent decrease in the frequency of resistance, as the population size of pre-existing RpoB mutants would be smaller due to limited expansion post-emergence. We chose glycerol, citrate and acetate to test our hypothesis in the soil organism Mycobacterium smegmatis, whose patterns of rifampicin resistance mirror those of $M t b^{40}$. As expected, these three carbon sources supported different growth rates with measured generation times of the wild type being $3.24 \mathrm{~h}\left({ }^{95 \%} \mathrm{CI}: 3.23-3.25 \mathrm{~h}\right.$ ), $6.17 \mathrm{~h}\left({ }^{95 \%} \mathrm{CI}: 6.09-6.25 \mathrm{~h}\right)$ and $17.62 \mathrm{~h}\left({ }^{95 \%} \mathrm{CI}: 17.61-17.62 \mathrm{~h}\right)$, respectively. We then determined the frequency of rifampicin resistance for bacteria grown on each carbon source using the Luria-Delbrück fluctuation assay. We found a striking correlation between carbon source and the calculated frequency of resistance, with bacteria grown in glycerol giving rise to rifampicin-resistant bacteria at a rate of $1.3 \times 10^{-8}\left({ }^{95^{\circ}} \mathrm{CI}: 1.2 \times 10^{-8}-1.5 \times 10^{-8}\right)$, those grown in 
citrate at a rate of $3.4 \times 10^{-9}\left({ }^{95 \%} \mathrm{CI}: 2.9 \times 10^{-9}-4.0 \times 10^{-9}\right)$ and acetate-cultured bacteria at a rate of $4.5 \times 10^{-10}\left({ }^{95 \%} \mathrm{CI}: 3.4 \times 10^{-10}-5.6 \times 10^{-10}\right)-$ see Figure $5 \mathrm{C}$. This trend was remarkable, because it showed that changing only the carbon source, keeping all other variables constant, could lead to a 28 -fold change in the frequency of resistance.

The disparity in outcomes between the two experimental approaches suggests that the availability of catabolic energy does not disproportionately influence the ability of RpoB mutants to survive. However, the impact of carbon source on the frequency of rifampicin-resistant bacteria within a population clearly suggests that carbon allocation might be an important driver of the fitness cost of rifampicin resistance.

\section{Discussion}

We normally expect that form follows function in bacteria: expression differences should reflect variations in physiological states. Indeed, we show that RpoB Ser450Leu imparted a measurable physiological perturbation in addition to conferring rifampicin resistance. Consistent with the suggested role of compensatory mutation ${ }^{18}$, we confirmed that in one strain, RpoC Leu516Pro reduced both, the apparent fitness cost of rifampicin resistance and the magnitude of the expression changes arising from it. However, we also showed that the nature of the perturbation was not consistent across different genetic backgrounds. Instead, we observed a strain-specific response to the RpoB mutation, both in terms of the relative impact on growth and the rearrangement of gene expression. We further observed that the magnitude of the fitness cost that RpoB Ser450Leu imposes on a strain was related to the overall increase in the resources allocated to the proteome. Based on these observations, we proposed the "Burden of expression" hypothesis, with which we posited that in $M t b$, the cost of rifampicin resistance was mediated by the metabolic burden imposed by the modified baseline protein expression of resistant strains. Elaborating on this hypothesis we demonstrated that interfering with anabolic processes could suppress the emergence of rifampicin resistance in the related organism M. smegmatis. 
The "Burden of expression" hypothesis stems from experimental data with clear caveats. First, we started our analyses assuming that ribosomal biosynthesis is unlikely to play a key role in the cost of rifampicin resistance in $M t b$ and that therefore expression data were a better window into the modified physiology. Our data seem to support the validity of this assumption: ribosomal proteins represented only $5.5 \%$, on average, of the total protein biomass in our experiments. This proportion was marginally higher in RpoB mutants, and it seemed to increase with increasing generation time (see Supplementary Figure 15). These trends were more consistent with a cost imposed by the metabolic burden of making ribosomes. Second, some of our key conclusions are based on a relatively small number of strains. Nonetheless, to the best of our knowledge, this sample set represents the most comprehensive and best curated account of rifampicin resistanceinduced global expression changes in $M t b$ to date, covering both: evolutionary dynamics and phylogenetic diversity. We were also able to show that patterns of expression detected in the DSRifR comparison were robust when the same strain pair was sampled again (see Figure 4 and Supplementary Figure 7). Importantly, key inferences that led us to propose the hypothesis came from SWATH-MS proteomic data drawn from the five different strain backgrounds. These data showed a clear clustering of biological replicates (see Supplementary Figure 16), with the exception of N0145 for which we were also unable to detect a significant cost for the Ser450Leu mutation or any significant changes to the expression. Third, we assumed that label free quantification (LFQ) using the "best flyer peptide" or TopN approach, which reflects the proportional abundance of individual proteins within our samples ${ }^{41}$, can be used to draw conclusions about the resource investment of the cell and can be extended to the growth rate of bacteria. It is possible that the roles are reversed and the growth rate of bacteria in fact determines the protein complement being expressed ${ }^{42}$. We addressed this possibility by performing a comparison of proteome investment and growth rate for wild type strains only. If the growth rate of $M t b$ did indeed determine the protein complement of cells across genetic distances on an evolutionary timescale, we would expect a strong correlation between differences 
in proteome and differences in growth rates between any two strains. This was however not the case (see Supplementary Figure 13). Finally, we also assumed that the proteome plays a central role in imposing a limit to the growth rate of an $M t b$ cell. There are other components that require considerable investment in carbon: in the case of $M t b$ both lipids and cell wall may act as a sink for resources limiting growth as they can account for over half of the dry mass of actively growing cells ${ }^{43}$. Lipidomic analysis of RpoB mutants in $M t b$ pointed to differences in mycobactin biosynthesis as one of the biggest discrepancies between rifampicin-resistant mutants and their susceptible ancestors $^{44}$. While echoing a key observation from our quest for determining the cost of resistance, we saw no evidence that mycobactin biosynthesis itself changes the rate of bacterial growth. The virulence-associated phthiocerol dimycocerosates (PDIM) have also been implicated in the cost of rifampicin resistance ${ }^{45}$, as have other changes in lipid composition ${ }^{46}$. The full exploration of the role of lipids in the physiology of rifampicin-resistant $M t b$ is beyond the scope of this study, but it would provide an interesting new and complementary avenue to pursue.

Keeping these considerations in mind, there are two striking features to emerge from our results. The first is the pervasive epistasis modulating the impact of RpoB Ser450Leu: the same mutation has markedly different effects on the physiology of different $M t b$ strains. The second is the apparent mechanism through which modulation of gene expression is propagated across the levels of bacterial physiology. Modification in RNAP function seems to have pleiotropic effects that transcend the disruption of any single group of genes, and impart a perturbation that appears to affect bacterial resource allocation.

One question that remains open is what sits at the heart of the disparity in phenotypes? The sequence of RNAP is effectively the same in all strains ${ }^{47}$; and by extension so are the biochemical changes that arise from resistance ${ }^{13}$. We envisage that part of the answer lays in differences in underlying robustness: a strain's capacity to buffer perturbation. Furthermore, we can consider this a window into the evolutionary adaptation of each strain and a sign of how different their physiologies really are. The amalgamation of mutational differences that effectively makes up a 
strain genetic background weaves a baseline phenotype that allows different $M t b$ strains to be successful pathogens despite differences in their underlying physiology. These differences are unmasked by the presence of a mutation that sits at the core of gene expression and reveals idiosyncratic transcriptional responses to rifampicin resistance that are poorly conserved across genetic distances. This observation has the implication that, beyond the described mutations in BBDP, which seem to alleviate some of the biochemical and gene expression effects of rifampicin resistance more generally, further investigation of positive selection of compensation of resistance-related traits should be performed in genetically related strains as they could vary considerably when comparing phylogenetically distant strains ${ }^{21,48}$.

The strain-specific nature of resistance-related expression perturbations can be used to provide a credible link to disparate growth rate modulation. Our suggestion that proteome composition influences growth rate is not without precedent. This connection has been made before ${ }^{49}$, and resulted in the formulation of a collection of "growth laws" that linked growth rates to the partitioning of the limited proteome between ribosomes and other proteins carrying out the rest of the cellular functions. Growth on different carbon sources impacted this balance, with "poorer" ones requiring a greater investment into the functional proteome, presumably because of the need for anabolic reactions increased the reliance on biosynthetic enzymes. A similar relationship has been observed in a wide range of microbial species ${ }^{50}$. An elaboration of these growth relationships also led to the conclusion that the efficiency of proteome allocation can impact growth rates and cell physiology ${ }^{51}$. Our finding that the increase in the relative cost of the proteome brought about by the gain of a mutation correlates with the relative fitness of that mutation is consistent with these reports, as is our observation that anabolic processes may play a mechanistic role in setting the cost of a mutation.

The observed differential cost of rifampicin resistance across $M t b$ strains, provides a lens through which we can better understand the emergence of drug resistance in clinical TB. However, it also indicates a new avenue to pursue in the fight against rifampicin resistant $M t b$ and perhaps 
uncover a new paradigm for chemotherapeutic intervention. Agents that impart a considerable shock to the expression equilibrium of bacteria could exhibit potent activity against rifampicin resistant strains due to collateral sensitivity. Furthermore, when given in combination with rifampicin, such agents may act to suppress the emergence of resistance; a valuable attribute for lengthening the shelf life of rifampicin.

\section{Methods}

\section{Strains and culture conditions}

We used four strains described by Comas $e t a l^{52}$ : namely the wild type, clinical isolate T85

(N0155, DS), a rifampicin resistant mutant of T85 carrying the Ser450Leu mutation (N1981, RifR), a derivative of T85 that was evolved by serial passage (200 generations) in the absence of rifampicin (N1588, $\mathrm{DS}^{\text {evo }}$ ) and an evolved derivative of the rifampicin resistant strains carrying an additional mutation in RpoC - Leu516Pro (N1589, RifR ${ }^{\text {evo }}$.

In addition to these strains we used four clinical isolates that are part of the recently compiled Reference set of $M t b$ clinical strains ${ }^{53}$ covering the genetic diversity of $M t b$. Two strains belonging to Lineage 1 (N0072, N0157) and two to Lineage 2 (N0052, N0145). We plated each of these strains on $7 \mathrm{H} 10$ plates containing $5 \mu \mathrm{g} / \mathrm{ml}$ Rifampicin, and picked colonies of spontaneous mutants. We checked the rifampicin-resistance conferring mutations using Sanger sequencing of the amplified RRDR region (Forward primer: TCGGCGAGCTGATCCAAAACCA, Reverse primer: ACGTCCATGTAGTCCACCTCAG, product size: 601 bp), and kept a Ser450Leu derivative of each clinical strain (N2027, N2030, N2495 and N1888, respectively).

Bacteria were cultured in 11 bottles containing large glass beads to avoid clumping and $100 \mathrm{ml}$ of media incubated at $37^{\circ} \mathrm{C}$ rotated continuously on a roller. Unless otherwise stated we used a modified 7H9 medium supplemented with $0.5 \% \mathrm{w} / \mathrm{v}$ pyruvate, $0.05 \% \mathrm{v} / \mathrm{v}$ tyloxapol, $0.2 \% \mathrm{w} / \mathrm{v}$ glucose, $0.5 \%$ bovine serum albumin (Fraction V, Roche) and $14.5 \mathrm{mM} \mathrm{NaCl}$. Compared to the 
usual composition of $7 \mathrm{H} 9$ we omitted glycerol, tween 80 , oleic acid and catalase from the medium. We added $10 \mu \mathrm{M}$ Hemin (Sigma) when supplementing growth medium with iron. We followed growth by measuring optical density at $600 \mathrm{~nm}\left(\mathrm{OD}_{600}\right)$.

Fluctuation assay experiments were performed using Mycobacterium smegmatis, $\mathrm{mc}^{2}$ 155. $M$. smegmatis was grown either in $10 \mathrm{ml}$ cultures within $50 \mathrm{ml}$ Falcon conical tubes in a shaker incubator $\left(37^{\circ} \mathrm{C}, 200 \mathrm{RPM}\right)$, or as $200 \mu \mathrm{l}$ aliquots within flat-bottomed 96 -well plates at $37^{\circ} \mathrm{C}$ and shaken at $200 \mathrm{rpm}$. We used unmodified $7 \mathrm{H} 9$ medium or medium where glycerol was replaced with citrate or acetate added at concentrations that matched the molarity of carbon.

\section{Data availability and data analysis}

All RNAseq data were deposited in the ArrayExpress repository of the European Bioinformatics Institute under the E-MTAB-7359 accession.

Unless otherwise stated, we preformed the analyses using Python 3.5.2 augmented with the following modules to provide additional functionality: Matplotlib (ver 2.0.0), Numpy (ver 1.12.1), Scipy (ver 0.19.0), Pandas (ver 0.20.1), statsmodels (ver 0.8.0), sklearn (ver 0.18.1), and netwrokX (ver 1.11).

\section{Fitness determination}

Mtb fitness was determined by comparative growth rate estimation. We grew bacteria as described and followed their growth by measuring $\mathrm{OD}_{600}$. We transformed the optical density measurements using logarithm base 2 and trimmed all early and late data points that did deviated from the linear correlation expected for exponential growth. Next, we fitted a linear mixed effect regression model to the data. Fitness cost was calculated as the resistance imposed deviation from wild type growth dynamics.

For M. smegmatis, we determined the growth rates by culturing bacteria as described above. We monitored the increase in $\mathrm{OD}_{600}$ using a Tecan M200 Pro Nanoquant at 20 min intervals. The 
data were $\log 2$-transformed, trimmed to retain only the portion of data pertinent to exponential growth and used for fitting a mixed effect linear regression model to estimate growth parameters.

\section{Transcriptional analysis with RNAseq}

We transferred a $40 \mathrm{ml}$ aliquot of bacterial culture in mid-log phase $(\mathrm{OD} 600=0.5 \pm 0.1)$ into a $50 \mathrm{ml}$ Falcon conical tube containing $10 \mathrm{ml}$ ice. We harvested the cells by centrifugation $(3,000 \times g$, $7 \mathrm{~min}, 4^{\circ} \mathrm{C}$ ), re-suspended the pellet in $1 \mathrm{ml}$ of RNApro solution (MP Biomedicals) and transferred the suspension to a Lysing matrix B tube (MP Biomedicals). We disrupted the bacterial cells using a FastPrep24 homogeniser (40s, intensity setting 6.0, MP Biomedicals). We clarified the lysate by centrifugation $\left(12,000 \times g, 5 \mathrm{~min}, 4^{\circ} \mathrm{C}\right)$, transferred the supernatant to a clean tube and added chloroform. We separated the phases by centrifugation $\left(12,000 \times g, 5 \mathrm{~min}, 4^{\circ} \mathrm{C}\right)$ and precipitated the nucleic acids from the aqueous phase by adding ethanol and incubating at 20C overnight. We performed a second acid phenol extraction to enrich for RNA. We treated our samples with DNAse I Turbo (Ambion), and removed stable RNAs by using the RiboZero Gram Positive ribosomal RNA depletion kit (Epicentre). We prepared the sequencing libraries using the TruSeq stranded Total RNA kit (Illumina) and sequenced on a HiSeq2500 high output run (50 cycles, single end).

Illumina short reads were mapped to the $M t b H 37 \mathrm{Rv}$ reference genome using BWA (ver 0.7.13); the resulting mapping files were processed with samtools (ver 1.3.1). Per-feature read counts were performed using the Python module htseq-count (ver 0.6.1p1) and Python (ver 2.7.11). We performed differential expression analysis using the R package DESeq2 ${ }^{54}$ (ver 1.16.1) and R (ver 3.4.0). In the case of the identification of the signature of compensation we performed a comparison of RifR vs DS $+\mathrm{DS}^{\text {evo }}+$ RifR $^{\text {evo }}$. For the follow-up experiments we performed two separate comparisons: $\left(\mathrm{DR}^{\mathrm{N} 0072}+\mathrm{DR}^{\mathrm{N} 0157}+\mathrm{DR}^{\mathrm{N} 0052}+\mathrm{DR}^{\mathrm{N} 0145}+\mathrm{DR}^{\mathrm{N} 0155}\right)$ vs $\left(\mathrm{DS}^{\mathrm{N} 0072}+\mathrm{DS}^{\mathrm{N} 0157}\right.$ $+\mathrm{DS}^{\mathrm{N} 0052}+\mathrm{DS}^{\mathrm{N} 0145}+\mathrm{DS}^{\mathrm{N} 0155}$ ) as well as individual DR vs DS comparisons. 
Gene set enrichment analysis was based on functional annotation from the Kyoto Encyclopaedia of Genes and Genomes and a custom collation of curated gene sets based on published reports.

The overrepresentation analysis was based on Fisher's exact as the discriminating test.

In addition we transformed per-feature counts into transcript counts per million bases (TPM).

TPM for each feature for each sample were calculated using the following formula:

$$
T P M_{i}=\frac{\frac{\text { counts }_{i}}{\text { size }_{i}}}{\sum_{j}^{n} \frac{\text { counts }_{j}}{\text { size }_{j}}}
$$

Where counts $s_{i}$ refers to the number of reads that map to a feature $i$, and size $_{i}$ refers to the length (in $\mathrm{bp)}$ of feature $i$. This ratio was normalized by dividing by the sum of all the ratios across all the features.

\section{Proteomic analysis with SWATH-MS}

We harvested $20 \mathrm{OD}_{600}$ equivalents from mid-log phase $\left(\mathrm{OD}_{600}=0.5 \pm 0.1\right)$ bacterial cultures by centrifugation $\left(3,000 \times g, 7 \mathrm{~min}, 4^{\circ} \mathrm{C}\right)$. We washed the bacterial pellet twice with phosphate buffered saline (PBS) to remove residues of tyloxapol. We re-suspended the bacterial pellet in 500 $\mu$ of protein lysis buffer (8M Urea, $0.1 \mathrm{M}$ Ammonium bicarbonate, $0.1 \%$ RapiGest [Waters]) and transferred the suspension to a Lysing matrix B tube (MP Biomedicals). We disrupted the bacterial cells using a FastPrep24 homogeniser (40s, intensity setting 6.0, MP Biomedicals). We clarified the lysate by centrifugation $\left(12,000 \times g, 5 \mathrm{~min}, 4^{\circ} \mathrm{C}\right)$, and sterilised the supernatant by passing it twice through a $0.22 \mu \mathrm{m}$ syringe filters (Milipore).

Following protein extraction for each sample, we used trypsin to digest proteins into peptides and then desalted them using $\mathrm{C}_{18}$ columns (The Nest Group). The cleaned up peptides were resuspended in MS buffer $(2 \% \mathrm{v} / \mathrm{v}$ acentonitrile, $0.1 \% \mathrm{v} / \mathrm{v}$ formic acid). Finally, the RT-kit (Biognosis) containing $11 \mathrm{iRT}$ retention time normalization peptides was spiked in to every sample. 
We measured every sample in sequential window acquisition of all theoretical mass spectra (SWATH) mode, a data independent acquisition implementation, on a triple'TOF 5600 mass spectrometer (AB Sciex) coupled to a nano flow HPLC system with the gradient of one hour ${ }^{55}$. The raw files acquired through a 64 variable width window precursor isolation scheme were centroid normalized using Proteowizard msconvert. We used the $M t b$ spectral library described earlier $^{56}$ to extract data using the OpenSWATH workflow ${ }^{57-59}$. The processed data were filtered by MAYU to $1 \%$ protein FDR ${ }^{60}$. R packages aLFQ and MSstats were used for protein quantification (Top3 peptides and top5 fragment ions ${ }^{41}$ ) and differential expression analysis respectively ${ }^{61,62}$.

\section{Mycobactin determination}

We harvested $5 \mathrm{OD}_{600}$ equivalents from mid-log phase $\left(\mathrm{OD}_{600}=0.5 \pm 0.1\right)$ bacterial cultures by centrifugation $\left(3,000 \times g, 7 \mathrm{~min}, 4^{\circ} \mathrm{C}\right)$. We washed the bacterial pellet three times with $15 \mathrm{ml}$ of cold, sterile $7 \mathrm{H} 9$ medium base devoid of additives (BD) to remove residues of tyloxapol. After washing we resuspended the pellets in $80 \mu \mathrm{l}$ of cold, sterile $7 \mathrm{H} 9$ medium base and added $750 \mu \mathrm{l}$ of 1:2 Chloroform:Methanol. We vortexed the samples for 5 minutes at top speed and added 750 $\mu \mathrm{l}$ of Chloroform. The samples were shaken for $1.5 \mathrm{~h}$ at room temperature and clarified by centrifugation $(16,000 \times g, 10 \mathrm{~min})$. We transferred the organic phase to a fresh tube, dried the samples in a speedvac and re-suspended each sample in $120 \mu$ of 44:44:2 Acetonitrile:Methanol:H2O, (v:v:v).

Chromatographic separation and analysis by mass spectrometry was done using a 1200 series HPLC system with a Phenomenex Kinetex column $(1.7 \mu \mathrm{l} \times 100 \mathrm{~mm} \times 2.1 \mathrm{~mm})$ with a SecurityGuard Ultra (Part No: AJ-9000) coupled to an Agilent Technologies 6550 Accurate-Mass Q-Tof. Solvent A: $\mathrm{H}_{2} \mathrm{O}, 10 \mathrm{mM}$ ammonium acetate; Solvent B: acetonitrile, $10 \mathrm{mM}$ ammonium acetate. $10 \mu \mathrm{l}$ of extract were injected and the column (C18) was eluted at $1.125 \mathrm{ml} / \mathrm{min}$. Initial conditions were $60 \%$ solvent B: 0-2 min, $95 \% \mathrm{~B} ; 2-4 \mathrm{~min}, 60 \% \mathrm{~B} ; 4-5 \mathrm{~min}$ at initial conditions. Spectra were collected in negative ion mode form $50-3200 \mathrm{mz}$. Continuous infusion of 
calibrants (Agilent compounds HP-321, HP-921, HP-1821) ensured exact masses over the whole mass range.

We converted the raw data files to the mzML format using msConvert and processed them in $\mathrm{R}$ using the $\mathrm{XCMS}^{63}$ (ver 3.0.2). We extracted targeted ion chromatograms with CAMERA (ver 1.34.0).

\section{Transcriptional module analysis.}

The iron-responsive sub-graph of the global gene regulation network published by Peterson et $a l^{28}$, was generated by using all expression modules and all iron-responsive genes as nodes, with edges connecting them representing module membership. All other gene nodes were discarded, keeping only the information pertinent to the number of genes present in each module (its degree). We focused explicitly on modules with at least 3 IdeR-dependent iron-responsive genes within them. Finally we marked significant differential expression of the gene nodes in every comparison.

For the purposes of contextualising the expressional profiling of RpoB Ser450Leu we selected a subset of expression modules as follows: first we collated all the genes that were differentially expressed in at least one genetic background as determined by pairwise comparisons. We then scored each expression module for enrichment of membership by differentially expressed genes using a binomial test. We retained all modules for which the test pointed to an excess of differentially regulated genes $(\mathrm{p}<0.05)$. We constructed a new sub-graph of the global regulatory network using all enriched modules and their constituent genes irrespective of whether or not individual genes were significantly differentially expressed. Edges reflected module membership. We added expression information in the form of log-fold changes of abundance to each subgraph based on pairwise analyses. 


\section{Calculation of genetic distance between clinical isolates}

Genetic distance between strains was defined as the number of single nucleotide variants (SNV) that separate two strains. The numeric value of this parameter was extracted from the phylogeny published elsewhere ${ }^{53}$.

\section{Quantification of the relative impact of the rpoB mutation on gene expression in}

\section{different clinical isolates}

We define the dissimilarity in the expressional response to the presence of the $r p o B$ mutation using three metrics: absolute number of shared significantly differentially expressed genes, the fraction of both the shared significantly differentially expressed genes and shared non-affected genes (hamming distance) and the Euclidean distance between ratios of TPM. The first is simply the number of shared genes that were found to be significantly affected by the presence of the $r p o B$ mutation in two different genetic backgrounds. For the second we use the same input to calculate the hamming distance between the patterns of genes significantly affected by the mutation in $r p o B$ in two different genetic backgrounds. In the third case we first calculate the TPM. We then calculate the mean TPM for each gene across the biological replicates as well as the ratio of mutant to wild type mean TPM for every gene. This gives us a vector containing 4000 ratios for each mutant-wild type pair. Finally we calculate the Euclidean distance between these vectors for the different genetic backgrounds. We plotted each of these metrics against genetic distance and calculated the spearman correlation and the coefficient of variance: standard deviation over mean multiplied by $100(\sigma / \mu \times 100 \%)$.

\section{Quantification of the absolute impact of the rpoB mutation on gene expression of a}

\section{clinical isolate}

We used transcript counts per million bases (TPM) and label free quantification (LFQ) to generate an RNA vector and a protein vector containing all the available information for each measured sample. We then calculated all the possible DS - RifR pairwise Euclidean distances for 
the RNA and protein vectors within each genetic background. We used the mean and standard deviation for the dissimilarity estimates. We evaluated the correlation between the fitness cost of RpoB mutations and the expression distance using the $\mathrm{R}^{2}$-coefficient derived from ordinary least squares linear regression as well as the Spearman correlation. Arbitrary units expressing the dissimilarity were obtained by dividing the calculated distances by 500,000 or $10,000,000$ for TPM and LFQ, respectively.

\section{Estimation of the biosynthetic cost of protein production}

The calculation of biosynthetic cost was based on the molecular weight of amino acids (MW) ${ }^{36}$ or on the estimate of E. coli ATP investment into individual amino acids derived by Akashi et al. ${ }^{37}$ using the following formulae:

$$
\begin{gathered}
p_{i}=L F Q_{i, X} \times \sum_{j=1}^{k} \alpha_{j}^{M W} \text { or } p_{i}=L F Q_{i, X} \times \sum_{j=1}^{k} \alpha_{j}^{A T P} \\
P_{X}=\sum_{i}^{n} p_{i}
\end{gathered}
$$

Where the cost of protein $i\left(p_{i}\right)$ was calculated as the sum of the cost for each constituent amino acid $\left(\alpha_{j}^{M W / A T P}\right)$ based either on its molecular weight $(M W)$ or ATP investment $(A T P)$ and adjusted by the proportional contribution of protein $i$ to the total proteome of sample $X\left(L F Q_{i, X}\right)$. The overall cost of the proteome $P$ for a sample $X\left(P_{X}\right)$ is expressed as the sum of the costs of individual proteins $(p)$. The difference between the biosynthetic investments in the proteome of sample X when compared to sample $\mathrm{Y}$ was simply: $P_{X}-P_{Y}$. We estimated the biosynthetic perturbation of RpoB Ser450Leu within a genetic background, by resampling sample-specific proteome costs for DS and RifR with replacement 100-times, and using the median as well as the $3^{\text {rd }}$ and $98^{\text {th }}$ quantiles to provide the $95 \%$ confidence interval. Finally, we quantify the correlation with the relative fitness of RpoB Ser450Leu by calculating the Spearman coefficient. 


\section{Minimum inhibitory concentration determination}

We used the microplate alamar blue assay ${ }^{64}$ to determine the minimum inhibitory concentrations of bedaquiline in all drug susceptible and drug resistant strains used in our study. We tested bedaquiline using a two-fold dilution series spanning a concentration of $4 \mathrm{ng} / \mathrm{ml}-1 \mu \mathrm{g} / \mathrm{ml}$.

\section{Fluctuation Assay for determining the frequency of rifampicin resistance}

We used the Luria-Delbrück fluctuation assay ${ }^{65}$ to determine the frequency of rifampicin resistance in Mycobacterium smegmatis. Briefly, we inoculated 30 parallel cultures containing $10 \mathrm{ml}$ of modified Middlebrook 7H9 medium containing either glycerol, citrate or acetate as the main carbon source with 5000 colony forming units of pre-adapted M. smegmatis. We grew the cultures to mid-log phase $\left(\mathrm{OD}_{600}=0.5\right)$ at which point we chose three cultures at random for the determination of overall population size. We harvested the remaining bacteria by centrifugation $4000 \times g$ for 7 minutes, re-suspended the cellular pellet with $500 \mu \mathrm{l}$ of fresh Middlebrook 7 H9 medium and plated onto Middlebrook 7H10 solid media supplemented with $200 \mu \mathrm{g} / \mathrm{ml}$ Rifampicin. Plates were incubated at $37^{\circ} \mathrm{C}$ for 3-4 days and scored by counting the resulting resistant colonies. We determined the population-wide number of mutants $(m)$ using an in house implementation of the Ma-Sandri-Sarkar maximum likelihood estimation ${ }^{66}$, and adjusted it by the estimated population size to determine the frequency of resistance.

\section{Acknowledgements}

Calculations were performed at sciCORE (http://scicore.unibas.ch/) scientific computing center at University of Basel, with support by the SIB - Swiss Institute of Bioinformatics. This work was supported by the SystemsX.ch project "TbX", the National Institutes of Health project Omics4TB Disease Progression (U19 AI106761), Swiss National Science Foundation (grants 310030_166687, IZRJZ3_164171, IZLSZ3_170834 and CRSII5_177163) and the European Research Council (309540-EVODRTB). The authors would like to thank Uwe Sauer and Michael Zimmermann for their input during the early stages of the project. 


\section{References}

1. Laxminarayan, R. et al. Access to effective antimicrobials: a worldwide challenge. I ancet 387, 168-75 (2016).

2. Review of Antimicrobial Resistance, Tackling Drug-Resistant Infections Globally: Final Report and Recommendations. (ed. O'Neill, J.) 84 (Wellcome Trust and UK Government, London, 2016).

3. Holmes, A.H. et al. Understanding the mechanisms and drivers of antimicrobial resistance. Lancet 387, 176-87 (2016).

4. Melnyk, A.H., Wong, A. \& Kassen, R. The fitness costs of antibiotic resistance mutations. Evol Appl 8, 273-83 (2015).

5. Andersson, D. \& Hughes, D. Effects of antibiotic resistance on bacterial fitness, viruelnce and transmission. in Evolutionary biology of bacterial and fungal pathogens (eds. Baquero, F., Nombela, C., Cassell, G.H. \& Gutierrez-Fuentes, J.A.) 307-318 (ASM Press, Washington, DC, 2008).

6. Campbell, E.A. et al. Structural mechanism for rifampicin inhibition of bacterial RNA polymerase. Cell 104, 901-12 (2001).

7. Thiele, I., Jamshidi, N., Fleming, R.M. \& Palsson, B.O. Genome-scale reconstruction of Escherichia colis transcriptional and translational machinery: a knowledge base, its mathematical formulation, and its functional characterization. PLoS Comput Biol 5, e1000312 (2009).

8. Gourse, R.L., Gaal, T., Bartlett, M.S., Appleman, J.A. \& Ross, W. rRNA transcription and growth rate-dependent regulation of ribosome synthesis in Escherichia coli. Annu Rev Microbiol 50, 645-77 (1996).

9. Qi, Q., Preston, G.M. \& MacLean, R.C. Linking system-wide impacts of RNA polymerase mutations to the fitness cost of rifampin resistance in Pseudomonas aeruginosa. MBio 5, e01562 (2014).

10. Reynolds, M.G. Compensatory evolution in rifampin-resistant Escherichia coli. Genetics 156, 1471-81 (2000).

11. Ehrenberg, M., Bremer, H. \& Dennis, P.P. Medium-dependent control of the bacterial growth rate. Biochimie 95, 643-58 (2013).

12. Song, T. et al. Fitness costs of rifampicin resistance in Mycobacterium tuberculosis are amplified under conditions of nutrient starvation and compensated by mutation in the beta' subunit of RNA polymerase. Mol Microbiol 91, 1106-19 (2014).

13. Stefan, M.A., Ugur, F.S. \& Garcia, G.A. Source of the Fitness Defect in RifamycinResistant Mycobacterium tuberculosis RNA Polymerase and the Mechanism of Compensation by Mutations in the beta' Subunit. Antimicrob Agents Chemother 62(2018).

14. World Health Organization, Global Tuberculosis Report 2017. 147 (World Health Organization, Geneva, 2017).

15. Gygli, S.M., Borrell, S., Trauner, A. \& Gagneux, S. Antimicrobial resistance in Mycobacterium tuberculosis: mechanistic and evolutionary perspectives. FEMS Microbiol Rev (2017).

16. Gagneux, S. et al. The competitive cost of antibiotic resistance in Mycobacterium tuberulosis. Science 312, 1944-6 (2006).

17. Grandjean, L. et al. Transmission of Multidrug-Resistant and Drug-Susceptible Tuberculosis within Households: A Prospective Cohort Study. PLaS Med 12, e1001843; discussion e1001843 (2015).

18. Comas, I. et al. Whole-genome sequencing of rifampicin-resistant Mycobacterium tuberculosis strains identifies compensatory mutations in RNA polymerase genes. Nat Genet 44, 10610 (2012). 
19. de Vos, M. et al. Putative compensatory mutations in the rpoC gene of rifampin-resistant Mycobacterium tuberculosis are associated with ongoing transmission. Antimicrob Agents Chemother 57, 827-32 (2013).

20. Casali, N. et al. Evolution and transmission of drug-resistant tuberculosis in a Russian population. Nat Genet 46, 279-86 (2014).

21. Farhat, M.R. et al. Genomic analysis identifies targets of convergent positive selection in drug-resistant Mycobacterium tuberculosis. Nat Genet 45, 1183-9 (2013).

22. Molodtsov, V., Scharf, N.T., Stefan, M.A., Garcia, G.A. \& Murakami, K.S. Structural basis for rifamycin resistance of bacterial RNA polymerase by the three most clinically important RpoB mutations found in Mycobacterium tuberculosis. Mol Microbiol 103, 1034-1045 (2017).

23. Jones, C.M. et al. Self-poisoning of Mycobacterium tuberulosis by interrupting siderophore recycling. Proc Natl Acad Sii US A 111, 1945-50 (2014).

24. Wells, R.M. et al. Discovery of a siderophore export system essential for virulence of Mycobacterium tuberculosis. PLoS Pathog 9, e1003120 (2013).

25. Reddy, P.V. et al. Disruption of mycobactin biosynthesis leads to attenuation of Mycobacterium tuberculosis for growth and virulence. J Infect Dis 208, 1255-65 (2013).

26. Rodriguez, G.M., Voskuil, M.I., Gold, B., Schoolnik, G.K. \& Smith, I. ideR, An essential gene in Mycobacterium tuberculosis: role of IdeR in iron-dependent gene expression, iron metabolism, and oxidative stress response. Infect Immun 70, 3371-81 (2002).

27. Minch, K.J. et al. The DNA-binding network of Mycobacterium tuberculosis. Nat Commun 6, 5829 (2015).

28. Peterson, E.J. et al. A high-resolution network model for global gene regulation in Mycobacterium tuberculosis. Nucleic Acids Res 42, 11291-303 (2014).

29. Rustad, T.R. et al. Mapping and manipulating the Mycobacterium tuberculosis transcriptome using a transcription factor overexpression-derived regulatory network. Genome Biol 15, 502 (2014).

30. Gagneux, S. Ecology and evolution of Mycobacterium tuberculosis. Nat Rev Microbiol 16, 202213 (2018).

31. Coscolla, M. \& Gagneux, S. Consequences of genomic diversity in Mycobacterium tuberculosis. Semin Immunol 26, 431-44 (2014).

32. Ford, C.B. et al. Mycobacterium tuberculosis mutation rate estimates from different lineages predict substantial differences in the emergence of drug-resistant tuberculosis. Nat Genet (2013).

33. Borrell, S. \& Gagneux, S. Infectiousness, reproductive fitness and evolution of drugresistant Mycobacterium tuberculosis. Int J Tuberc Lung Dis 13, 1456-1466 (2009).

34. Park, H.D. et al. Rv3133c/dosR is a transcription factor that mediates the hypoxic response of Mycobacterium tuberculosis. Mol Microbiol 48, 833-43 (2003).

35. Zaneveld, J.R., McMinds, R. \& Vega Thurber, R. Stress and stability: applying the Anna Karenina principle to animal microbiomes. Nat Microbiol 2, 17121 (2017).

36. Seligmann, H. Cost-minimization of amino acid usage. J Mol Evol 56, 151-61 (2003).

37. Akashi, H. \& Gojobori, T. Metabolic efficiency and amino acid composition in the proteomes of Escherichia coli and Bacillus subtilis. Proc Natl Acad Sci US A 99, 3695-700 (2002).

38. Andries, K. et al. A diarylquinoline drug active on the ATP synthase of Mycobacterium tuberculosis. Science 307, 223-7 (2005).

39. Ycart, B. Fluctuation analysis: can estimates be trusted? PLoS One 8, e80958 (2013).

40. Borrell, S. et al. Epistasis between antibiotic resistance mutations drives the evolution of extensively drug-resistant tuberculosis. Evolution, Medicine, and Public Health eot003, 65-74 (2013).

41. Schubert, O.T. et al. Absolute Proteome Composition and Dynamics during Dormancy and Resuscitation of Mycobacterium tuberculosis. Cell Host Microbe 18, 96-108 (2015). 
42. Beste, D.J. et al. Transcriptomic analysis identifies growth rate modulation as a component of the adaptation of mycobacteria to survival inside the macrophage. $J$ Bacteriol (2007).

43. Beste, D.J. et al. Compiling a molecular inventory for Mycobacterium boris BCG at two growth rates: evidence for growth rate-mediated regulation of ribosome biosynthesis and lipid metabolism. J Bacteriol 187, 1677-84 (2005).

44. Lahiri, N. et al. Rifampin Resistance Mutations Are Associated with Broad Chemical Remodeling of Mycobacterium tuberculosis. J Biol Chem 291, 14248-56 (2016).

45. Bisson, G.P. et al. Upregulation of the phthiocerol dimycocerosate biosynthetic pathway by rifampin-resistant, rpoB mutant Mycobacterium tuberculosis. J Bacteriol 194, 6441-52 (2012).

46. du Preez, I. \& Loots du, T. Altered fatty acid metabolism due to rifampicin-resistance conferring mutations in the rpoB Gene of Mycobacterium tuberculosis: mapping the potential of pharmaco-metabolomics for global health and personalized medicine. OMICS 16, 596603 (2012).

47. Borrell, S. \& Trauner, A. Strain Diversity and the Evolution of Antibiotic Resistance. $A d v$ Exp Med Biol 1019, 263-279 (2017).

48. Zhang, H. et al. Genome sequencing of 161 Mycobacterium tuberculosis isolates from China identifies genes and intergenic regions associated with drug resistance. Nat Genet 45, 125560 (2013).

49. Scott, M., Gunderson, C.W., Mateescu, E.M., Zhang, Z. \& Hwa, T. Interdependence of cell growth and gene expression: origins and consequences. Science 330, 1099-102 (2010).

50. Karpinets, T.V., Greenwood, D.J., Sams, C.E. \& Ammons, J.T. RNA:protein ratio of the unicellular organism as a characteristic of phosphorous and nitrogen stoichiometry and of the cellular requirement of ribosomes for protein synthesis. BMC Biol 4, 30 (2006).

51. Basan, M. et al. Overflow metabolism in Escherichia coli results from efficient proteome allocation. Nature 528, 99-104 (2015).

52. Comas, I. et al. Human T cell epitopes of Mycobacterium tuberculosis are evolutionarily hyperconserved. Nat Genet 42, 498-503 (2010).

53. Borrell, S. et al. Reference Set of Mycobacterium tuberculosis Clinical Strains: A tool for research and product development. Preprint on bioRxiv: bttps:// mmw.biorxiv:org/content/ early/2018/08/25/399709.

54. Love, M.I., Huber, W. \& Anders, S. Moderated estimation of fold change and dispersion for RNA-seq data with DESeq2. Genome Biol 15, 550 (2014).

55. Banaei-Esfahani, A., Nicod, C., Aebersold, R. \& Collins, B.C. Systems proteomics approaches to study bacterial pathogens: application to Mycobacterium tuberulosis. Curr Opin Microbiol 39, 64-72 (2017).

56. Schubert, O.T. et al. The Mtb Proteome Library: A Resource of Assays to Quantify the Complete Proteome of Mycobacterium tuberculosis. Cell Host Microbe 13, 602-612 (2013).

57. Reiter, L. et al. mProphet: automated data processing and statistical validation for largescale SRM experiments. Nat Methods 8, 430-5 (2011).

58. Rost, H.L. et al. OpenSWATH enables automated, targeted analysis of data-independent acquisition MS data. Nat Biotechnol 32, 219-23 (2014).

59. Rost, H.L. et al. OpenMS: a flexible open-source software platform for mass spectrometry data analysis. Nat Methods 13, 741-8 (2016).

60. Reiter, L. et al. Protein identification false discovery rates for very large proteomics data sets generated by tandem mass spectrometry. Mol Cell Proteomics 8, 2405-17 (2009).

61. Rosenberger, G., Ludwig, C., Rost, H.L., Aebersold, R. \& Malmstrom, L. aLFQ: an Rpackage for estimating absolute protein quantities from label-free LC-MS/MS proteomics data. Bioinformatics 30, 2511-3 (2014).

62. Choi, M. et al. MSstats: an R package for statistical analysis of quantitative mass spectrometry-based proteomic experiments. Bioinformatics 30, 2524-6 (2014). 
63. Smith, C.A., Want, E.J., O'Maille, G., Abagyan, R. \& Siuzdak, G. XCMS: processing mass spectrometry data for metabolite profiling using nonlinear peak alignment, matching, and identification. Anal Chem 78, 779-87 (2006).

64. Franzblau, S.G. et al. Rapid, low-technology MIC determination with clinical Mycobacterium tuberculosis isolates by using the microplate Alamar Blue assay. J Clin Microbiol 36, 362-6 (1998).

65. Luria, S.E. \& Delbruck, M. Mutations of bacteria from virus sensitivity to virus resistance. Genetics 28, 491-511 (1943).

66. Sarkar, S., Ma, W.T. \& Sandri, G.H. On fluctuation analysis: a new, simple and efficient method for computing the expected number of mutants. Genetica 85, 173-9 (1992). 


\section{Figure Legends}

Figure 1: Conceptual workflow. A. Two complementary strain sets used for the experiments. Strains comprised in the "Evolutionary trajectory of rifampicin resistance" set were derived from a single clinical isolate (DS, N0155) by isolation of a Ser450Leu mutant in the lab and the subsequent passage for 200 generations in the absence of rifampicin. These strains were used to identify expression changes that are reversed by compensation - signature of compensation. The generalizability of our finding was checked using the "Genetic diversity strain set" containing five independent clinical isolates and their rifampicin-resistant derivatives. All rifampicin resistant strains shared the same resistance mutation - RpoB Ser450Leu. B. Experimental outline for the sampling and analyses.

Figure 2: Signature of compensation. A. The relative fitness of drug resistant strains (DR) is expected to be lower than wild type (DS) at first, but then is expected to increase due to compensatory evolution. The phenotypic equivalent of this trend is illustrated as an increase/decrease in a measurable trait upon the emergence of resistance that is then returned to its previous level through compensation. We refer to this dynamic as the "Signature of Compensation". B. Plot of transcript counts per million bases (TPM) and label free quantifications (LFQ) of cellular proteins for genes whose expression is perturbed by the Ser450Leu mutation in RpoB and returned to wild type in the presence of the compensating Leu516Pro mutation. All results were standardized across measurements for a single gene to allow the comparison between strains. Grey traces show genes that are significantly more highly expressed in RifR, yellow traces show genes that were significantly less highly expressed in RifR. The red and blue bold lines show the median of the sample for more and less highly expressed proteins, respectively.

Figure 3: RifR has a higher baseline level of mycobactin biosynthesis than DS. A. Subset of the gene regulatory network ${ }^{28}$ containing iron responsive genes. Circles represent IdeR-regulated genes that are either induced (black inner circle) or repressed (white inner circle) in low iron conditions. Hexagons represent IdeR-independent iron responsive genes that are induced (white inner hexagons) or repressed (black inner hexagons) in low iron conditions. We used blue and red to indicate significantly lower or higher RNA expression in RifR, respectively. Diamonds represent transcriptional modules as defined by Petersen et al, black diamonds indicate modules that contain at least 3 IdeRresponsive genes. Edges connect gene nodes with the module nodes they belong to. Labels 1-7 refer to Module 502 (1), Module 525 (2), Module 267 (3), Module 446 (4), Module 231 (5), Module 086 (6) and Module 295 (7) from the original publication. B. Relative mycobactin levels expressed as maximum peak heights for DS and RifR in normal medium (grey dots) and iron-supplemented medium $(10 \mu \mathrm{M}$ hemin, red dots). Unfilled circles represent the mean of the observations. 
Figure 4: The prominent role of mycobactin biosynthesis in the signature of compensation is not universal. A. Iron-responsive subset the of gene regulatory network, as shown in Figure 3, coloured based on transcriptional differential expression data from pairwise comparison of genetically distinct rifampicin-susceptible clinical isolates and their cognate RpoB Ser450Leu mutants. RifR and N0155 refer to an independent sampling of the same strain pairs. See Supplementary Figure 7 for the proteome counterpart of this plot. B. Representation of the enrichment of significantly differentially expressed genes within individual transcriptional modules, as defined by Peterson et al. ${ }^{28}$. The columns alternate proteomic $(P)$ and transcriptomic data $(R)$. "ALL" refers to the global differential expression analysis of all rifampicin-susceptible against all rifampicin-resistant strains. The remaining column annotations refer to individual pair-wise comparisons in different genetic backgrounds. Black squares represent no significant enrichment, mauve squares and yellow squares show enrichment at $0.01<p<0.05$ and $p<0.01$ using a Fisher's exact test. These $p$-values are not adjusted for multiple testing. Modules covering the DosR-regulon and IdeR-iron repressed regulon are highlighted separately.

Figure 5: The fitness cost of RpoB Ser450Leu correlates with increased resource requirements. A. The relative fitness of Ser450Leu RpoB mutants estimated from growth rate data is negatively correlated with the magnitude of the deviation from the resources allocated to the wild type proteome. es - Spearman correlation. B. Comparison of minimum inhibitory concentrations (MIC) of bedaquiline in clinical isolates and their cognate RpoB mutants. Dotted line shows parity, darker shading includes $50 \%$ or lower difference in MIC and the lighter shading spans up to 2-fold change in MIC. C. The frequency of rifampicin resistance as measured in the model organism Mycobacterium smegmatis with the Luria-Delbrück fluctuation assay is plotted against the generation time in media containing different carbon sources. 
A Strain set: Evolutionary trajectory of rifampicin resistance

Strain set: Genetic diversity

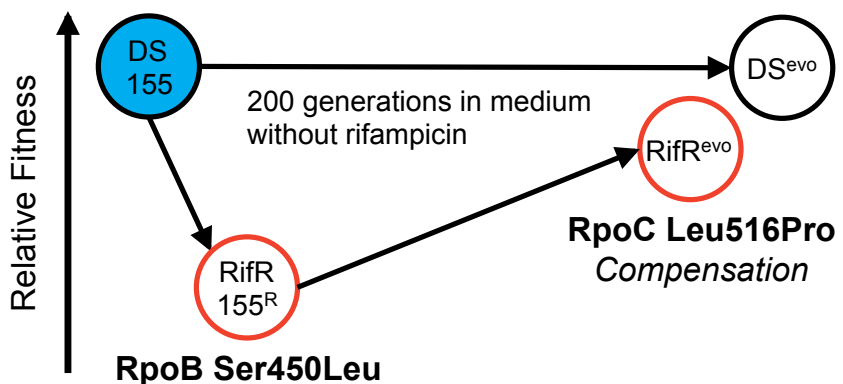

Unrelated Clinical isolates

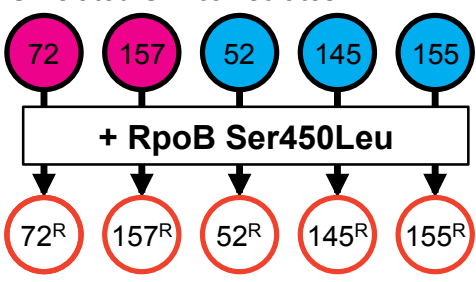

Rifampicin-resistant derivatives
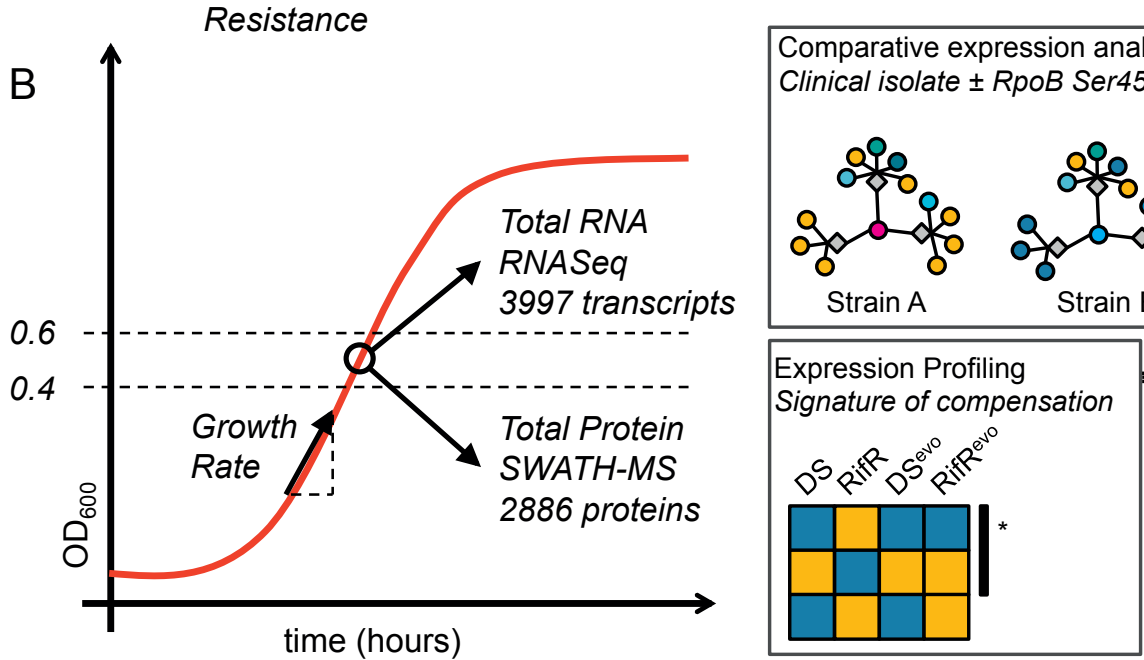

Resource Allocation Burden of expression

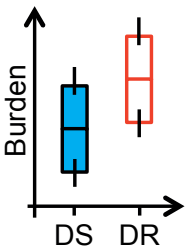

Experimental Validation

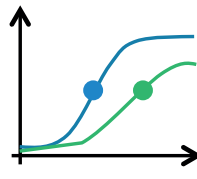

Phenotypes

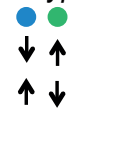


A
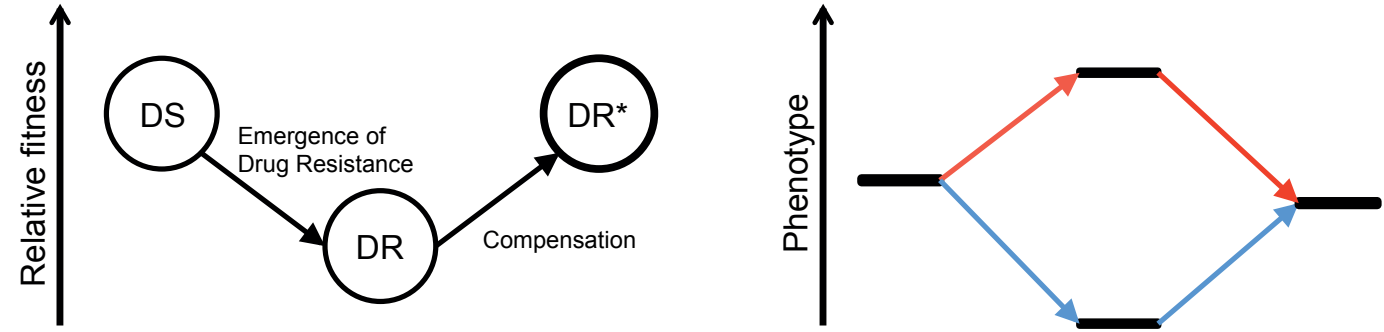

B
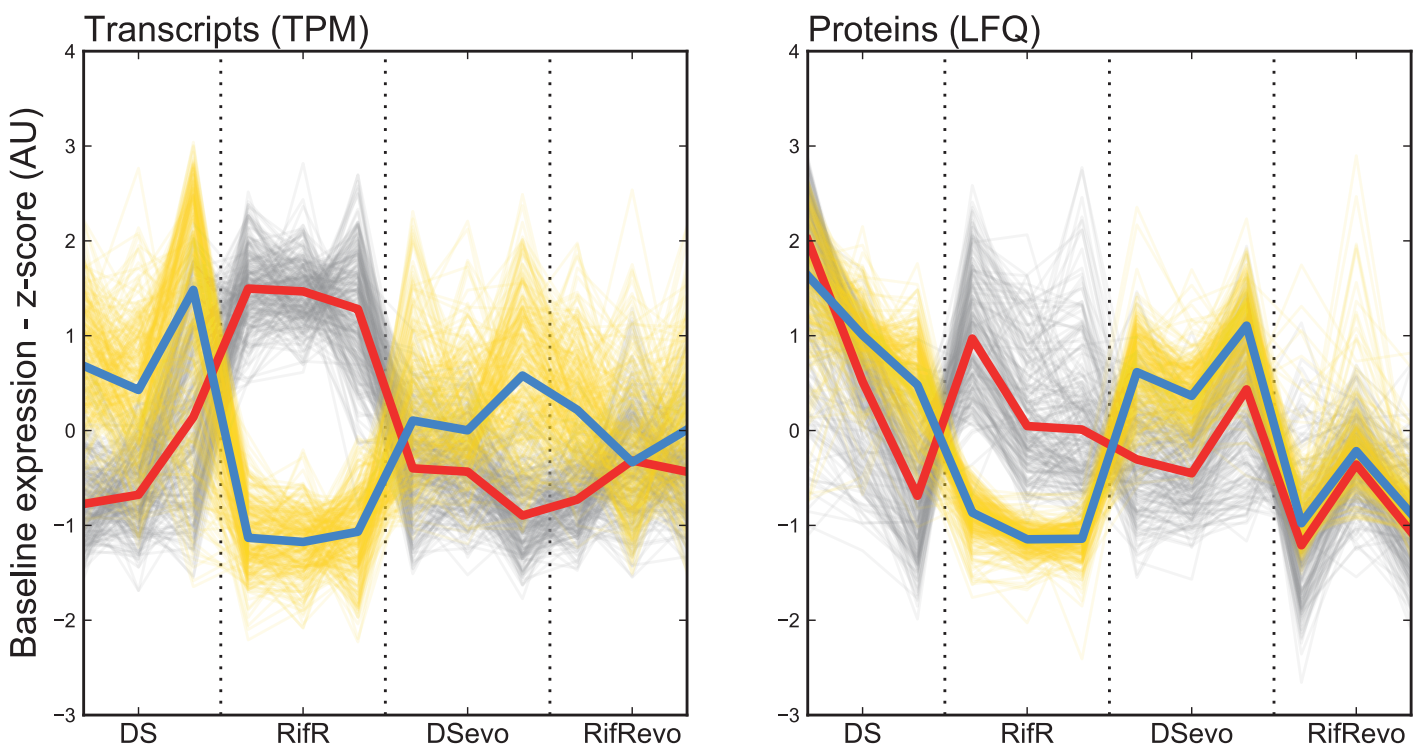


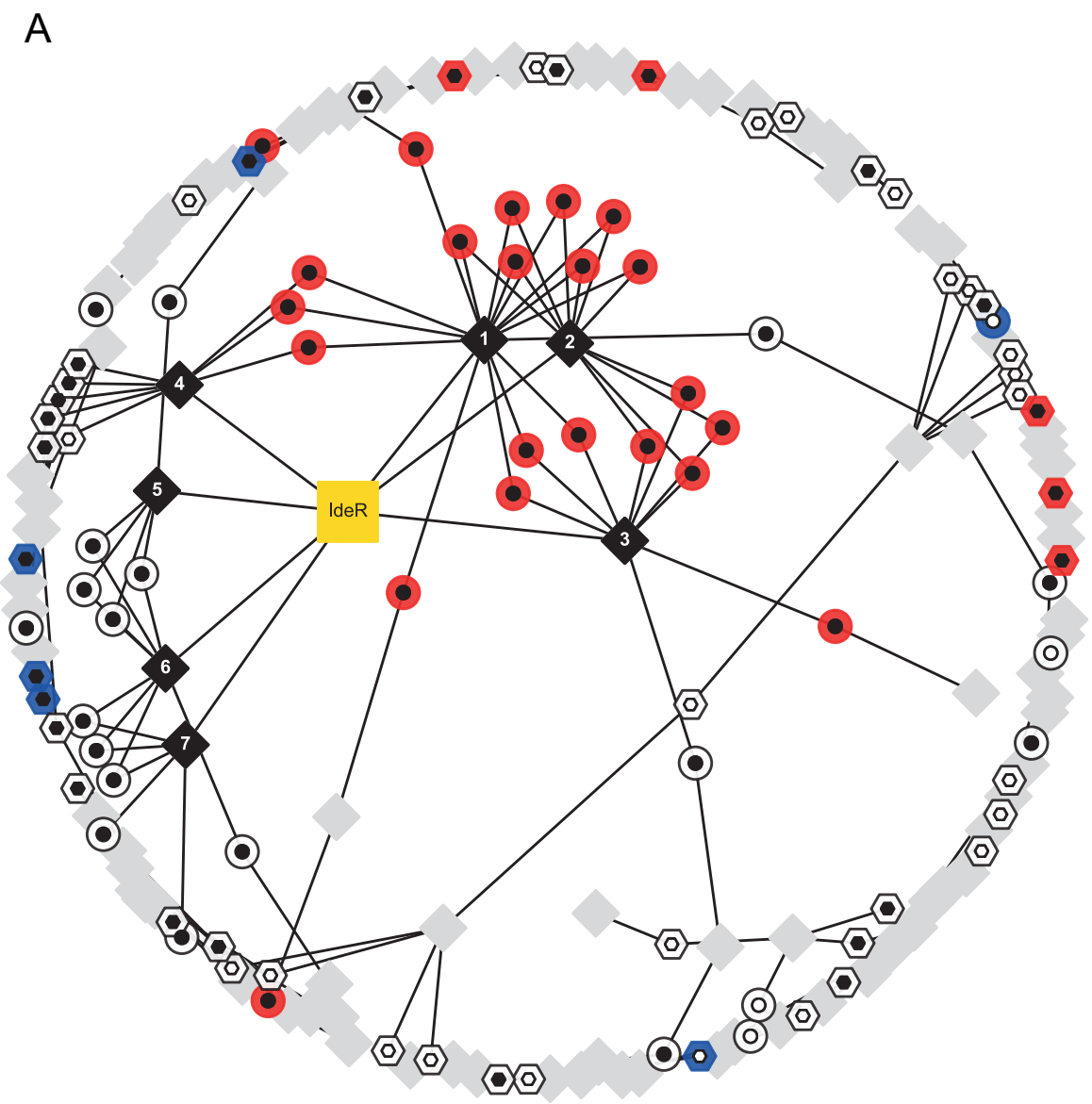

B

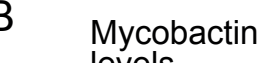
levels

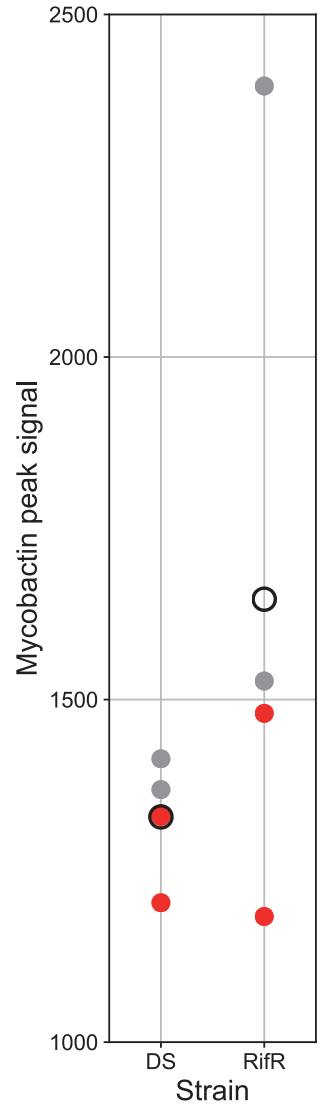


A RifR

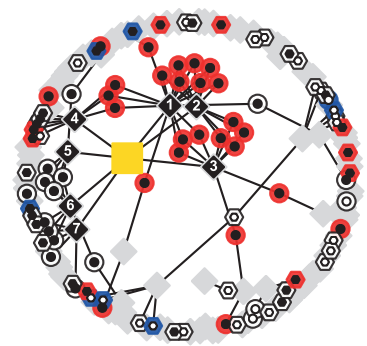

N0052

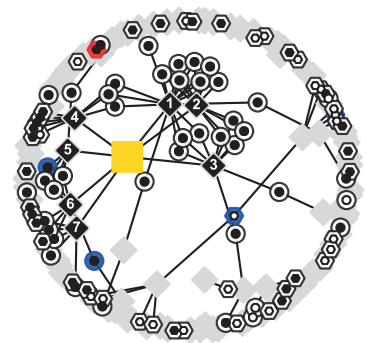

N0072

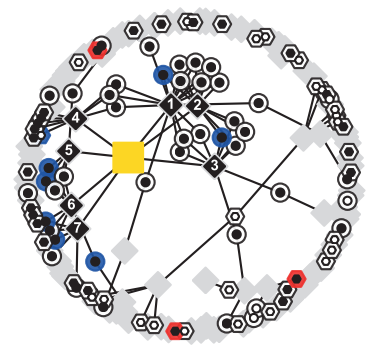

N0155

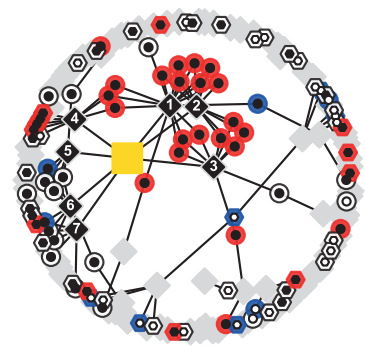

N0145

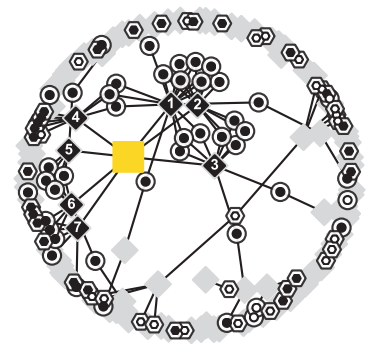

N0157

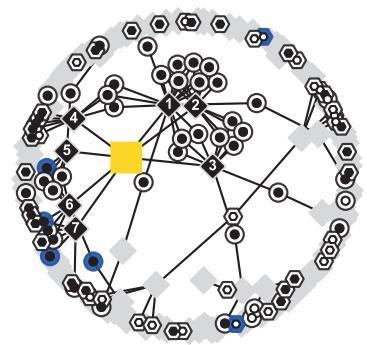

B

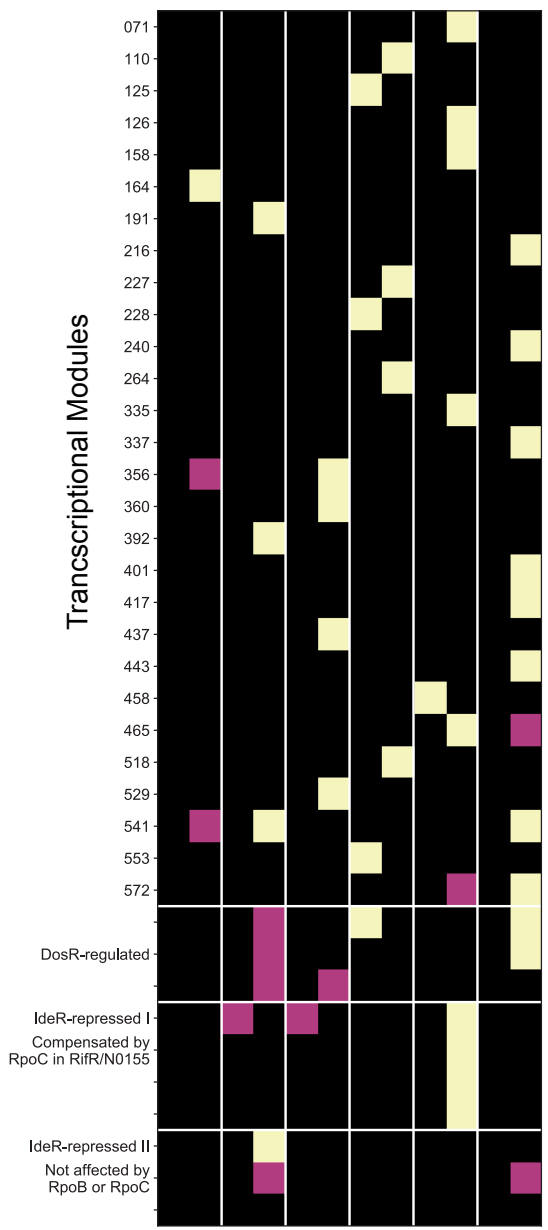


